\title{
Exploration of Genetic Diversity for Qualitative Traits among the Extant Upland Cotton (Gossypium hirsutum L.) Varieties and Parental Lines
}

\author{
K. Rathinavel* \\ Central Institute for Cotton Research, Regional Station, Coimbatore-641003, T.N., India \\ *Corresponding author
}

\section{A B S T R A C T}

A study was conducted to characterize and evaluate the extant varieties and parental lines of upland cotton using qualitative morphological traits for DUS testing of cotton. These data were subjected to various genetic principles. The results indicated that out of 26 traits,

\section{Keywords}

Qualitative traits,

Principal component analysis, Dendrogram, Eigenvalue.

\section{Article Info}

Accepted:

21 June 2017

Available Online:

10 August 2017 only 17 traits were included for Principal Component analysis (PCA) because of expression of variability. Traits like stem hairiness, flower petal colour and density of seed fuzz expressed much variation (four states) compared to other traits. Nine traits viz., hypocotyl pigmentation, leaf gossypol glands, leaf nectaries, leaf petiole pigmentation, stem pigmentation, male sterility, boll bearing habit, boll prominence of tip and boll opening showed no variability. The PCA revealed that the first seven PCs having eigen values $>1$ and contributed for $76.67 \%$ of the total variation. The variance explained by PC1 was mostly due to traits related to colour such as boll colour, petal colour, petal spot, anther filament colour, pollen colour, boll surface and leaf shape, whereas PC II mostly related to stem hairiness, leaf hairiness, leaf shape and bract type. Two-dimensional scatter plot was drawn supported with grouping patterns of cluster analysis. The cluster analysis classified 101 extant varieties into five divergent groups. The cluster II comprising of largest (57) genotypes followed by cluster I (36). The Shannon-Weaver diversity index (H') was used for estimating the frequency distribution of diversity index. The traits viz., hypocotyl pigmentation, leaf gossypol glands, leaf nectarines, leaf petiole pigmentation, stem pigmentation, flower male sterility, boll bearing habit, boll prominence of tip and boll opening were higher (4.62) when compared to other traits.

\section{Introduction}

Cotton is the leading non-food agricultural and industrial fibre crop grown in more than 80 countries (Dutt et al., 2004; Shakeel et al., 2011). Cotton is the world's most important natural textile fibre and an important source of feed, foodstuff, and oil, with approximate world consumption put at 27 million metric tons per year (Chen et al., 2007).

However, yield and quality of this allimportant crop have declined over the last decade due to huge general erosion in genetic diversity of cotton varieties (Meredith, 2000). Worldwide cotton breeders and producers have expressed concern over the narrow genetic basis of cultivated cotton germplasm that has caused a decline in yield and quality. Globally cotton breeding programmes are working with a narrow germplasm pool thus resulting in genetic bottleneck through historic domestication events and selection (Iqbal et al., 1997). Characterization of germplasm plays a vital role in crop improvement. Characterization, evaluation of 
germplasm and quantification of genetic diversity of genotypes is indispensable for a pragmatic use of plant genetic resources and also for determining evolutionary relationships (Zada et al., 2013).

The present study was envisaged to characterize extant varieties of cotton genotypes collected across the Indian subcontinent for qualitative traits using correlation analysis, principal component analysis, cluster analysis and diversity analysis. Knowledge of the associations among genotypes would help to identify a set of genotypes that have maximal diversity for the analysis of the effects of genetic background. Hierarchical cluster analysis has been suggested for classifying entries of germplasm collections based on degree of similarity and dissimilarity (Van Hintum 1995). Among these biometrical procedures, the main edge of principal component analysis (PCA) is that each genotype can be assigned to only one group and it also reflects the significance of the largest contributor to the total variability at each axis of differentiation (Sharma 1998) Genetic variation for morphological traits among the extant cotton varieties has been estimated using principal component analysis, which has led to the recognition of phenotypic variability (Sarvannan et al., 2006; Esmail et al., 2008; Li et al., 2008).

The objective of the present study was to evaluate the genetic diversity among cotton genotypes specifically to select the best genotypes by using PCA and cluster analysis that can be exploited in future cotton breeding programme was emanated from the data generated for characterisation and documentation of extant cotton varieties, a mandatory of Distinctiveness, Uniformity and Stability testing under The Protection of Plant Varieties and Farmers' Rights Act, 2001 of India which encourages stimulation and development of new plant varieties.

\section{Materials and Methods}

The investigation was carried out at Central Institute of Cotton Research, Regional Station, Coimbatore during 2012-14. The experimental material consisted of 101 extant cotton varieties and parental lines of hybrids, collected from diversified agro ecological cotton growing zones of India. Sowing of seeds of each variety was taken up in randomised block design with three replications. Each variety was sown in 10 rows adopting spacing of $90 \mathrm{~cm} \times 60 \mathrm{~cm}$. Recommended agronomic, cultural and plant protection practices for tetraploid cotton was adopted till the harvest of crop.

Qualitative characters were taken under consideration for evaluating the substantial variation and relationship among extant cotton genotypes. The ten plants were randomly selected and the observations recorded for 26 qualitative traits. The scores for hypocotyl pigmentation (HP) was given as 1- Absent, 9- Present; for leaf colour (LC) the rating given was 1- Light green, 2-Green, 3Light red and 4-Dark red; Leaf Hairiness (LH) was scored as 5- Medium, 9- Dense; Similarly Leaf Appearance (LA) 1-Cup, 2Flat; Leaf Gossypol Glands (LGG) as 1Absent, 9- Present; Leaf Nectaries (LN) 1Absent, 9- Present; Leaf petiole pigmentation (LPP) 1- Absent, 9- Present; Leaf shape (LS) 1- Palmate, 2- Semi-digitate, 3-Digitate, 4Lanceolate; Plant stem hairiness (SH) 1smooth, 3- sparse, 5- medium, 7-dense; plant stem pigmentation (SP) 1-Absent, 2-Present; Bract type (BT): 3- Normal, 5-Frego; Flower petal colour (FPC): 1-Cream, 2-Yellow, 3Deep yellow, 4-purple; Flower petal spot (FPS): 1-Absent, 9-Present; Flower stigma(FS): 3-Embedded, 5-Exerted; Flower anther filament colouration (FAFC): 1Absent, 9- Present; Flower pollen colour (FPoC): 1-White, 2- Cream, 3-Yellow, 4Deep yellow, 5- Purple; Flower male sterility (FMS): 1-Absent, 9-Present; Boll bearing 
habit (BBH): 1- Solitary, 9- Cluster; Boll Colour (BC): 3-Green, 5-Red; Boll shape (BSh): 3-Round, 5-Ovate, 7-Elliptic; Boll Surface (BS): 1-Smooth, 9-Pitted; Boll Prominence of tip (BPT): 1- Blunt, 9-Pointed; Boll opening (BO): 3- Semi-open, 5- Open; Seed fuzz (SF): 1-Naked, 3-Sparse, 5Medium, 7-Dense; Seed fuzz colour (SFC): 1White, 2-Grey, 3-Green, 4-Brown; Fibre colour (FC): 1-White, 2-Cream, 3-Green, 4Brown; were used for this study. Scores were allocated for each trait as per the National test guide lines of Protection of plant varieties and Farmer's Right's Authority of India (plantauthority.gov.in/)

Pearson correlation coefficient was worked out for the above 26 qualitative traits and correlation matrix was prepared for comparing different traits using XLSTAT version 2016.05 software. The standardized values of 26 qualitative traits were used to perform Principal Component Analysis (PCA) using PAST 3 (Hammer et al., 2001) to find out the relative importance of different traits and to capture the existing genetic variation. A screen plot was drawn from the Eigen values associated with a component or factor in descending order versus the number of the component or factor. Screen plot usually used for visual assessment of components or factor which explain much of the variability in the data. Shannon-Weaver diversity index (H') (Shannon and Weaver 1949) was arrived and used as a measure of phenotypic diversity of each trait. A low H' index indicates extreme unbalance frequency classes for an individual traits and lack of genetic diversity. Out of 26 traits, 9 traits have not expressed for genetic variability among the extant genotypes and the remaining 17 corresponding Principal component factors were subjected to cluster analysis based on Euclidean distances and wards minimum variance using Agglomerative hierarchical clustering method through XLSTAT 2016. A hierarchical cluster analysis for pooled data was performed using scores of dissimilarity matrix as per Ward 1963.

\section{Results and Discussion}

\section{Evaluation of genotypes based on qualitative traits}

The observations on 26 qualitative traits were recorded for all the 101 genotypes and analysed as per scores presented in table 1. The genotypes have expressed for green leaf colour (98) followed by light red (2) and one genotype dark red colour (BN red). For leaf hairiness, the highest number of genotypes is of medium (93) class followed by sparse (6) and dense (2). Majority of the genotypes were observed with flat nature of leaf (78) and rest of them is cup type (23). Three classes of leaf shape namely palmate, semi digitate and digitate were observed. Maximum numbers of genotypes (97) were observed with palmate leaf shape, while two genotypes namely $70 \mathrm{E}$ and GSAV 1056 are semi digitate (semi-okra) and another two genotypes viz., MDH 89 and SRT-GMS 1 were digitate (Okra). Stem hairiness recorded was medium in 73 genotypes followed by dense (16), sparse (5) and one with smooth type (GSB 39). All the genotypes were observed with normal bract type (100) except MDH 89 with frego bract. Cream petal colour was predominant (68) followed by yellow (29), purple (3) and one genotype with deep yellow petal colour (GSB 39). Flower petal spot was absent in 98 genotypes and were present in AK 32, GSAV 1056 and GSB 39. The embedded position of stigma was recorded in most of the genotypes (68) and in the remaining 33 genotypes it was exerted. Absence of anther filament colouration was observed in all the genotypes except GSAV 1056. Maximum number of genotypes had cream pollen colour (77) followed by yellow (21) and deep yellow (3). 
Boll colour namely green and red were observed of this green coloured bolls were found high 99 genotypes and red colour bolls appeared in BN red and GSAV 1056. Ovate boll shape (93) was observed in higher number of genotypes than round (6) and elliptic (2). Among the smooth and pitted boll surface, smooth was found in 98 genotypes and pitted recorded in GSB 39, SRT-GMS1 and GSAV 1056. The density of seed fuzz observed in maximum genotypes was medium (64) followed by dense (27), sparse (8). The genotypes BN red and GSB 39 did not bear seed fuzz and were naked. Grey seed fuzz colour (89) was found predominant followed by white (12). The fiber colour observed was cream in 94 genotypes when compared to white in the rest (7).

Among the 26 qualitative traits studied, the traits like stem hairiness, flower petal colour and seed fuzz showed higher amount of variation (four groups), while other traits showed only two or three category. The traits like hypocotyl pigmentation, leaf gossypol glands, leaf nectaries, leaf petiole pigmentation, plant stem pigmentation, male sterility, boll bearing, prominence of boll tip, boll opening habit showed no variation. Kumar et al., (2013) distinguished the genotypes based on flower petal colour in cluster bean. Flower petal colour aids removal of off-type plants, contaminants there by maintenance of genetic purity of seed production plots.

It was reported in cotton, that hybrids were successfully recognized and distinguished by morphological markers such as flower colour, spot position and their colours in petal, fibre colour, seed linter, leaf colour and their shapes (Hosseini 2014). Therefore, above qualitative traits will be very much useful for identifying true hybrids in hybridization programme.

\section{Correlation}

Correlation matrix was calculated from the standardized data of only 17 qualitative traits, since other nine characters such as hypocotyl pigmentation, leaf gossypol glands, leaf nectaries, leaf petiole pigmentation, plant stem pigmentation, male sterility, boll bearing habit, boll prominence of tip and boll opening were excluded due to lack of variability.

Correlation coefficient (Pearson 1901), among the 17 qualitative traits brought out the significant positive correlation of leaf colour with boll colour, pollen colour and flower petal colour; however, negatively correlated with density of seed fuzz. The trait leaf hairiness has positive significance with stem hairiness, boll shape and density of seed fuzz. The highly positive significant correlation was observed between the traits leaf appearance with seed fuzz colour; leaf shape with stem hairiness, bract type, flower petal colour, flower anther filament colouration, flower pollen colour, boll colour and boll surface. The stem hairiness has positive association with density of seed fuzz. The flower petal colour was positively correlated with flower petal spot, anther filament colour, pollen colour and boll surface whereas negatively correlated with fibre colour. Flower petal spot exhibited a positive correlation with position of stigma, anther filament colour, pollen colour, boll colour, boll shape and boll surface.

This was negatively correlated with seed fuzz colour. The positive association of position of stigma with boll colour; anther filament colour with boll colour and boll surface; pollen colour with boll colour, boll shape and boll surface; boll colour with boll surface; boll shape with boll surface were observed. Fibre colour showed negative correlation with leaf shape, pollen colour, petal colour, anther filament colour and boll surface (Table 2). 


\section{Principal Component Analysis (PCA)}

The maintenance and exploitation of genetic resources could be made possible by partitioning the total variance into its components.

It also provides a chance for utilization of appropriate genetic resource in crop improvement for a particular plant trait (Sneath and Sokal 1973; Pecetti et al., 1996). The Principal Component Analysis divides the total variance into different factors which is considered as a powerful tool to obtain parental lines for a successful breeding programme (Akter et al., 2009).
To discern patterns of variation, PCA was performed on all variables simultaneously. In this study, seven significant principal components (PCs) extracted had Eigen value $>1$ and this seven values accounted for a cumulative variation of $76.67 \%$. However, the remaining ten components contributed only 23.33\% towards the total diversity for this set of cotton genotypes. Among the seven PCs which has Eigen value $>1$, the first principal component (PC I) explained the most variability accounted for $23.87 \%$, followed by $11.85 \%, 10.76 \%, 8.42 \%, 8.21 \%, 7.05 \%$ $6.52 \%$, the remaining components respectively in the ascending order for total variation (Table 3 ).

Table.1 Table of frequency distribution of qualitative characteristics for tetraploid cotton (Gossypium hirsutum L)

\begin{tabular}{|c|c|c|c|c|c|}
\hline Sl. No. & Descriptors & Scores & states & $\begin{array}{c}\text { Number of } \\
\text { genotypes }\end{array}$ & $\begin{array}{c}\text { Frequency } \\
(\%)\end{array}$ \\
\hline \multirow{2}{*}{1} & \multirow{2}{*}{ Hypocotyl: Pigmentation } & 1 & Absent & - & - \\
\hline & & 9 & Present & 101 & 100 \\
\hline \multirow{4}{*}{2} & \multirow{4}{*}{ Leaf: Colour } & 1 & Light green & - & - \\
\hline & & 2 & Green & 98 & 97.03 \\
\hline & & 3 & Light red & 2 & 1.98 \\
\hline & & 4 & Dark red & 1 & 0.99 \\
\hline \multirow{3}{*}{3} & \multirow{3}{*}{ Leaf: Hairiness } & 1 & Sparse & 6 & 5.94 \\
\hline & & 5 & Medium & 93 & 92.08 \\
\hline & & 9 & Dense & 2 & 1.98 \\
\hline \multirow{2}{*}{4} & \multirow{2}{*}{ Leaf: Appearance } & 1 & Cup & 23 & 22.77 \\
\hline & & 2 & Flat & 78 & 77.23 \\
\hline \multirow{2}{*}{5} & \multirow{2}{*}{ Leaf: Gossypol glands } & 1 & Absent & - & - \\
\hline & & 9 & Present & 101 & 100 \\
\hline \multirow{2}{*}{6} & \multirow{2}{*}{ Leaf: Nectaries } & 1 & Absent & - & - \\
\hline & & 9 & Present & 101 & 100 \\
\hline \multirow{2}{*}{7} & \multirow{2}{*}{$\begin{array}{l}\text { Leaf: Petiole } \\
\text { pigmentation }\end{array}$} & 1 & Absent & - & - \\
\hline & & 9 & Present & 101 & 100 \\
\hline \multirow{4}{*}{8} & \multirow{4}{*}{ Leaf: Shape } & 1 & Palmate (Normal) & 97 & 96.04 \\
\hline & & 2 & Semi-digitate (Semi- Okra) & 2 & 1.98 \\
\hline & & 3 & Digitate (Okra) & 2 & 1.98 \\
\hline & & 4 & Lanceolate (Super Okra) & - & - \\
\hline \multirow{4}{*}{9} & \multirow{4}{*}{ Plant: Stem hairiness } & 1 & Smooth & 1 & 0.99 \\
\hline & & 3 & Sparse & 5 & 4.95 \\
\hline & & 5 & Medium & 79 & 78.22 \\
\hline & & 7 & Dense & 16 & 15.84 \\
\hline
\end{tabular}




\begin{tabular}{|c|c|c|c|c|c|}
\hline \multirow{2}{*}{10} & \multirow{2}{*}{ Plant: Stem pigmentation } & 1 & Absent & - & - \\
\hline & & 9 & Present & 101 & 100 \\
\hline \multirow{2}{*}{11} & \multirow{2}{*}{ Bract: Type } & 3 & Normal & 100 & 99.01 \\
\hline & & 5 & Frego & 1 & 0.99 \\
\hline \multirow{4}{*}{12} & \multirow{4}{*}{ Flower: Petal colour } & 1 & Cream & 68 & 67.33 \\
\hline & & 2 & Yellow & 29 & 28.71 \\
\hline & & 3 & Deep Yellow & 1 & 0.99 \\
\hline & & 4 & Purple & 3 & 2.97 \\
\hline \multirow{2}{*}{13} & \multirow{2}{*}{ Flower: Petal spot } & 1 & Absent & 98 & 97.03 \\
\hline & & 9 & Present & 3 & 2.97 \\
\hline \multirow{2}{*}{14} & \multirow{2}{*}{ Flower: Stigma } & 3 & Embedded & 68 & 67.33 \\
\hline & & 5 & Exerted & 33 & 32.67 \\
\hline \multirow{2}{*}{15} & \multirow{2}{*}{$\begin{array}{l}\text { Flower: Anther Filament } \\
\text { colouration }\end{array}$} & 1 & Absent & 100 & 99.01 \\
\hline & & 9 & Present & 1 & 0.99 \\
\hline \multirow{5}{*}{16} & \multirow{5}{*}{ Flower: Pollen colour } & 1 & White & - & - \\
\hline & & 2 & Cream & 77 & 76.24 \\
\hline & & 3 & Yellow & 21 & 20.79 \\
\hline & & 4 & Deep Yellow & 3 & 2.97 \\
\hline & & 5 & Purple & - & - \\
\hline \multirow{2}{*}{17} & \multirow{2}{*}{ Flower: Male Sterility } & 1 & Absent & 101 & 100 \\
\hline & & 9 & Present & - & - \\
\hline \multirow{2}{*}{18} & \multirow{2}{*}{ Boll: Bearing habit } & 1 & Solitary & 101 & 100 \\
\hline & & 9 & Cluster & - & - \\
\hline \multirow{2}{*}{19} & \multirow{2}{*}{ Boll: Colour } & 3 & Green & 99 & 98.02 \\
\hline & & 5 & Red & 2 & 1.98 \\
\hline \multirow{3}{*}{20} & \multirow{3}{*}{$\begin{array}{l}\text { Boll: Shape (longitudinal } \\
\text { section) }\end{array}$} & 3 & Round & 6 & 5.94 \\
\hline & & 5 & Ovate & 93 & 92.08 \\
\hline & & 7 & Elliptic & 2 & 1.98 \\
\hline \multirow{2}{*}{21} & Boll. Surface & 1 & Smooth & 98 & 97.03 \\
\hline & Borl: Surrace & 9 & Pitted & 3 & 2.97 \\
\hline 22 & Boll- Prominence of tin & 1 & Blunt & - & - \\
\hline 22 & Boll: Prominence of tip & 9 & Pointed & 101 & 100 \\
\hline 23 & Boll: Opening & 3 & Semi-open & - & - \\
\hline & & 5 & Open & 101 & 100 \\
\hline & & 1 & Naked & 2 & 1.98 \\
\hline 24 & Seed. Furz & 3 & Sparse & 8 & 7.92 \\
\hline 24 & Seed: FUZZ & 5 & Medium & 64 & 63.37 \\
\hline & & 7 & Dense & 27 & 26.73 \\
\hline & & 1 & White & 12 & 11.88 \\
\hline 25 & Seed. Fuzz colour & 2 & Grey & 89 & 88.12 \\
\hline 25 & seed. FUZZ corour & 3 & Green & - & - \\
\hline & & 4 & Brown & - & - \\
\hline & & 1 & White & 7 & 6.93 \\
\hline 26 & Fibre' Colour & 2 & Cream & 94 & 93.07 \\
\hline 20 & Fibre: Colour & 3 & Green & - & - \\
\hline & & 4 & Brown & - & - \\
\hline
\end{tabular}


Table.2 Correlation among qualitative traits in Gossypium hirsutum L. cotton genotypes

\begin{tabular}{|c|c|c|c|c|c|c|c|c|c|c|c|c|c|c|c|c|c|}
\hline & $\mathrm{LC}$ & $\mathrm{LH}$ & LA & $\mathrm{LS}$ & $\mathrm{SH}$ & BT & FPC & FPS & FS & FAFC & FPOC & $\mathrm{BC}$ & $\mathrm{BSH}$ & BS & SF & SFC & $\mathrm{FC}$ \\
\hline $\mathrm{LC}$ & 1.000 & 0.023 & -0.107 & -0.032 & 0.054 & -0.016 & 0.275 & -0.029 & 0.149 & -0.016 & 0.320 & 0.568 & 0.023 & -0.029 & -0.298 & -0.194 & 0.045 \\
\hline $\mathrm{LH}$ & & 1.000 & -0.077 & 0.027 & 0.607 & 0.014 & -0.022 & -0.184 & -0.128 & 0.014 & -0.065 & 0.020 & 0.235 & -0.184 & 0.201 & 0.058 & 0.101 \\
\hline LA & & & 1.000 & 0.028 & -0.335 & 0.054 & -0.068 & -0.183 & -0.226 & 0.054 & -0.180 & 0.077 & -0.077 & -0.044 & 0.015 & 0.238 & -0.055 \\
\hline LS & & & & 1.000 & 0.227 & 0.628 & 0.321 & 0.155 & 0.071 & 0.304 & 0.215 & 0.203 & 0.027 & 0.533 & -0.045 & 0.071 & -0.326 \\
\hline $\mathrm{SH}$ & & & & & 1.000 & 0.186 & 0.013 & -0.032 & 0.132 & 0.186 & -0.096 & 0.119 & 0.026 & -0.151 & 0.308 & 0.004 & -0.030 \\
\hline BT & & & & & & 1.000 & -0.060 & -0.017 & 0.144 & -0.010 & -0.053 & -0.014 & 0.014 & -0.017 & -0.023 & 0.037 & 0.027 \\
\hline FPC & & & & & & & 1.000 & 0.424 & 0.157 & 0.394 & 0.424 & 0.560 & 0.139 & 0.601 & -0.140 & -0.150 & -0.249 \\
\hline FPS & & & & & & & & 1.000 & 0.251 & 0.572 & 0.369 & 0.394 & 0.234 & 0.656 & -0.224 & -0.116 & -0.182 \\
\hline FS & & & & & & & & & 1.000 & 0.144 & 0.133 & 0.204 & 0.099 & 0.127 & -0.096 & -0.070 & 0.107 \\
\hline FAFC & & & & & & & & & & 1.000 & 0.145 & 0.704 & 0.014 & 0.572 & -0.023 & 0.037 & -0.366 \\
\hline FPOC & & & & & & & & & & & 1.000 & 0.347 & 0.216 & 0.485 & -0.124 & -0.290 & -0.241 \\
\hline BC & & & & & & & & & & & & 1.000 & 0.020 & 0.394 & -0.257 & -0.167 & -0.241 \\
\hline BSH & & & & & & & & & & & & & 1.000 & 0.234 & -0.023 & -0.162 & 0.101 \\
\hline BS & & & & & & & & & & & & & & 1.000 & -0.224 & -0.116 & -0.412 \\
\hline SF & & & & & & & & & & & & & & & 1.000 & -0.107 & 0.064 \\
\hline SFC & & & & & & & & & & & & & & & & 1.000 & 0.141 \\
\hline $\mathrm{FC}$ & & & & & & & & & & & & & & & & & 1.000 \\
\hline
\end{tabular}

Values in bold are significant at 5\%, LC Leaf Colour; LH Leaf Hairiness; LA Leaf Appearance; LS Leaf shape; SH Stem hairiness; BT bract type; FPC Flower petal colour; FPS Flower petal spot; FS Flower stigma; FAFC Flower Anther Filament Colouration; FPOC Flower Pollen colour; BC Boll Colour; BS Boll Shape; BS Boll Surface; SF Seed Fuzz; SFC Seed Fuzz Colour; FC Fibre Colour 
Table.3 Principal Components with Eigen values >1.0 for various traits in Gossypium hirsutum L. cotton genotypes

\begin{tabular}{|c|c|c|c|c|c|c|c|}
\hline \multirow{2}{*}{ Characters } & \multicolumn{7}{|c|}{ Principal Components } \\
\hline & PC1 & PC2 & $\mathbf{P C 3}$ & PC4 & PC5 & PC6 & PC7 \\
\hline Boll shape & 0.837 & -0.124 & 0.245 & -0.266 & 0.117 & 0.000 & 0.105 \\
\hline Boll Colour & 0.749 & 0.010 & -0.168 & 0.365 & -0.412 & 0.070 & -0.010 \\
\hline Flower Petal Colour & 0.745 & -0.090 & 0.060 & 0.010 & -0.112 & -0.060 & 0.090 \\
\hline Flower Petal Spot & 0.719 & -0.107 & -0.010 & -0.300 & 0.131 & 0.377 & -0.020 \\
\hline Flower Anther Filament Colour & 0.704 & 0.153 & 0.267 & -0.070 & -0.387 & 0.327 & -0.118 \\
\hline Flower Pollen Colour & 0.613 & -0.107 & -0.293 & -0.101 & 0.164 & -0.354 & 0.090 \\
\hline Fibre Colour & -0.457 & 0.010 & -0.346 & 0.169 & 0.242 & 0.416 & 0.264 \\
\hline Stem Hairiness & 0.010 & 0.893 & -0.127 & 0.010 & -0.163 & 0.080 & -0.168 \\
\hline Leaf Hairiness & -0.123 & 0.724 & -0.193 & -0.060 & -0.314 & 0.000 & 0.385 \\
\hline Leaf Shape & 0.491 & 0.402 & 0.544 & 0.207 & 0.317 & -0.250 & 0.050 \\
\hline Leaf Appearance & -0.120 & -0.285 & 0.498 & 0.229 & -0.337 & -0.070 & 0.402 \\
\hline Leaf Colour & 0.303 & -0.060 & -0.582 & 0.623 & -0.150 & -0.200 & 0.050 \\
\hline Seed Fuzz & -0.297 & 0.426 & -0.030 & -0.444 & -0.152 & -0.170 & -0.205 \\
\hline Bract Type & 0.030 & 0.421 & 0.400 & 0.439 & 0.545 & -0.141 & 0.050 \\
\hline Seed Fuzz Colour & -0.234 & 0.030 & 0.465 & 0.241 & -0.144 & 0.498 & 0.238 \\
\hline Flower Stigma & 0.292 & 0.119 & -0.257 & 0.186 & 0.410 & 0.473 & -0.287 \\
\hline Boll Shape & 0.214 & 0.156 & -0.280 & -0.344 & 0.290 & 0.060 & 0.685 \\
\hline Eigen values & 4.06 & 2.02 & 1.83 & 1.43 & 1.40 & 1.20 & 1.11 \\
\hline Variability (\%) & 23.87 & 11.85 & 10.76 & 8.42 & 8.21 & 7.05 & 6.52 \\
\hline Cumulative (\%) & 23.87 & 35.72 & 46.48 & 54.90 & 63.11 & 70.15 & 76.67 \\
\hline
\end{tabular}


Table.4 Grouping of Gossypium hirsutum L. cotton genotypes based on qualitative traits

\begin{tabular}{|c|c|c|}
\hline Clusters & No. of genotypes & Genotypes \\
\hline I & 36 & $\begin{array}{l}\text { 70 E, AB 6, AC738, ACP 71, AH 107, AK 32, AKH } 07 \text { R, Bikaneri Nerma, Ca/H- } \\
\text { 128, Ca/ H- 129, Ca/ MH -133, MDH 89, CPD 423, CSH -19, DHY 286 - 1, DS } 28 \\
\text { F, M 12, MCU 13, T 7, VC 21, VC 22, VC 31, J 34, Khandwa 3, F 1378, G cot. } 10 \text {, G } \\
\text { cot. 16, G cot. 100, PIL 43, RHC 006, KH 11, PG 5, PG 6, K 35, L 604, LRA 5166 }\end{array}$ \\
\hline II & 57 & $\begin{array}{l}\text { AK } 23 \text { B, CPD 420, CPD 428, CSH -8, G COT 18, GUJARAT 67, H 1098, H } 1117 \text {, } \\
\text { H } 1157 \text {, HLS 329, BN Red, LRA } 5166 \text { (SB), M 2, MCU 3, MCU 4, MCU 7, MCU 8, } \\
\text { NCH 11, NH 452, PH 325, PH 93, PUSA 8-6, REBA B 50, RHC 003, RS -2013, RS- } \\
\text { 810, RS-875, RST -9, Suman, TCH 1218, H 1220, VC 32, Vikas, Vikram, H 974, } \\
\text { HHM -1, F 286, LH 1134, F 320, LH 372, J 205, LSS, LH 886, Badnawar 1, GSHV } \\
\text { 112, HS 6, AKH 081, Deviraj, F 1054, F 1861, F 505, F 846, LH 1556, PIL 8, LH } \\
\text { 900, Surat Dwarf, Khandwa 2 }\end{array}$ \\
\hline III & 5 & K 34007, Kanchana, PRS 74, SH - 2379, L 389 \\
\hline IV & 2 & GSB 39, SRT-GMS1, \\
\hline $\mathrm{V}$ & 1 & GSAV 1056 \\
\hline
\end{tabular}

Table.5 Mean values of clusters for various qualitative traits in

Gossypium hirsutum L. tetraploid cotton

\begin{tabular}{|l|c|c|c|c|c|}
\hline Variable & Cluster I & Cluster II & Cluster III & Cluster IV & Cluster V \\
\hline HP & 9.00 & 9.00 & 9.00 & 9.00 & 9.00 \\
\hline LC & 2.04 & 2.05 & 2.00 & 2.00 & 2.00 \\
\hline LH & 5.16 & 5.00 & 1.00 & 3.00 & 5.00 \\
\hline LA & 1.64 & 1.91 & 2.00 & 1.50 & 2.00 \\
\hline LGG & 9.00 & 9.00 & 9.00 & 9.00 & 9.00 \\
\hline LN & 9.00 & 9.00 & 9.00 & 9.00 & 9.00 \\
\hline LPP & 9.00 & 9.00 & 9.00 & 9.00 & 9.00 \\
\hline LS & 1.06 & 1.00 & 1.00 & 2.00 & 2.00 \\
\hline SH & 5.60 & 4.95 & 3.40 & 3.00 & 7.00 \\
\hline SP & 9.00 & 9.00 & 9.00 & 9.00 & 9.00 \\
\hline BT & 3.04 & 3.00 & 3.00 & 3.00 & 3.00 \\
\hline FPC & 1.42 & 1.23 & 1.20 & 3.50 & 4.00 \\
\hline FPS & 1.16 & 1.00 & 1.00 & 5.00 & 9.00 \\
\hline FS & 4.04 & 3.14 & 3.80 & 4.00 & 5.00 \\
\hline FAFC & 1.00 & 1.00 & 1.00 & 1.00 & 9.00 \\
\hline FPC & 2.26 & 2.21 & 2.00 & 4.00 & 3.00 \\
\hline FMS & 1.00 & 1.00 & 1.00 & 1.00 & 1.00 \\
\hline BBH & 1.00 & 1.00 & 1.00 & 1.00 & 1.00 \\
\hline BC & 3.00 & 3.05 & 3.00 & 3.00 & 5.00 \\
\hline BS & 4.96 & 4.95 & 3.80 & 6.00 & 5.00 \\
\hline BS & 1.00 & 1.00 & 1.00 & 9.00 & 9.00 \\
\hline BPT & 9.00 & 9.00 & 9.00 & 9.00 & 9.00 \\
\hline BO & 5.00 & 5.00 & 5.00 & 5.00 & 5.00 \\
\hline SF & 6.08 & 4.58 & 4.60 & 3.00 & 5.00 \\
\hline SFC & 1.82 & 1.95 & 2.00 & 1.50 & 2.00 \\
\hline FC & 1.98 & 1.93 & 1.80 & 1.50 & 1.00 \\
\hline
\end{tabular}

Table.6 Shannon-Weaver Diversity Indices $\left(\mathrm{H}^{\prime}\right)$ of different traits

\begin{tabular}{|c|c|c|c|c|c|c|c|c|c|c|c|}
\hline Characters & HP & LC & LH & LA & LGG & LN & LPP & LS & SH & SP & BT \\
\hline H' & 4.62 & 4.61 & 4.58 & 4.58 & 4.62 & 4.62 & 4.62 & 4.59 & 4.60 & 4.62 & 4.61 \\
\hline Characters & FPC & FPS & FS & FAFC & FPC & FMS & BBH & BC & BS & BS & BPT \\
\hline H' & 4.52 & 4.35 & 4.58 & 4.51 & 4.59 & 4.62 & 4.62 & 4.61 & 4.61 & 4.35 & 4.62 \\
\hline Characters & BO & SF & SFC & FC & Average & S.d & & & & & \\
\hline H' & 4.62 & 4.58 & 4.60 & 4.61 & 4.58 & 0.07 & & & & & \\
\hline
\end{tabular}


Fig.1 Scatter plot based on PC1 and PC2 of qualitative characters

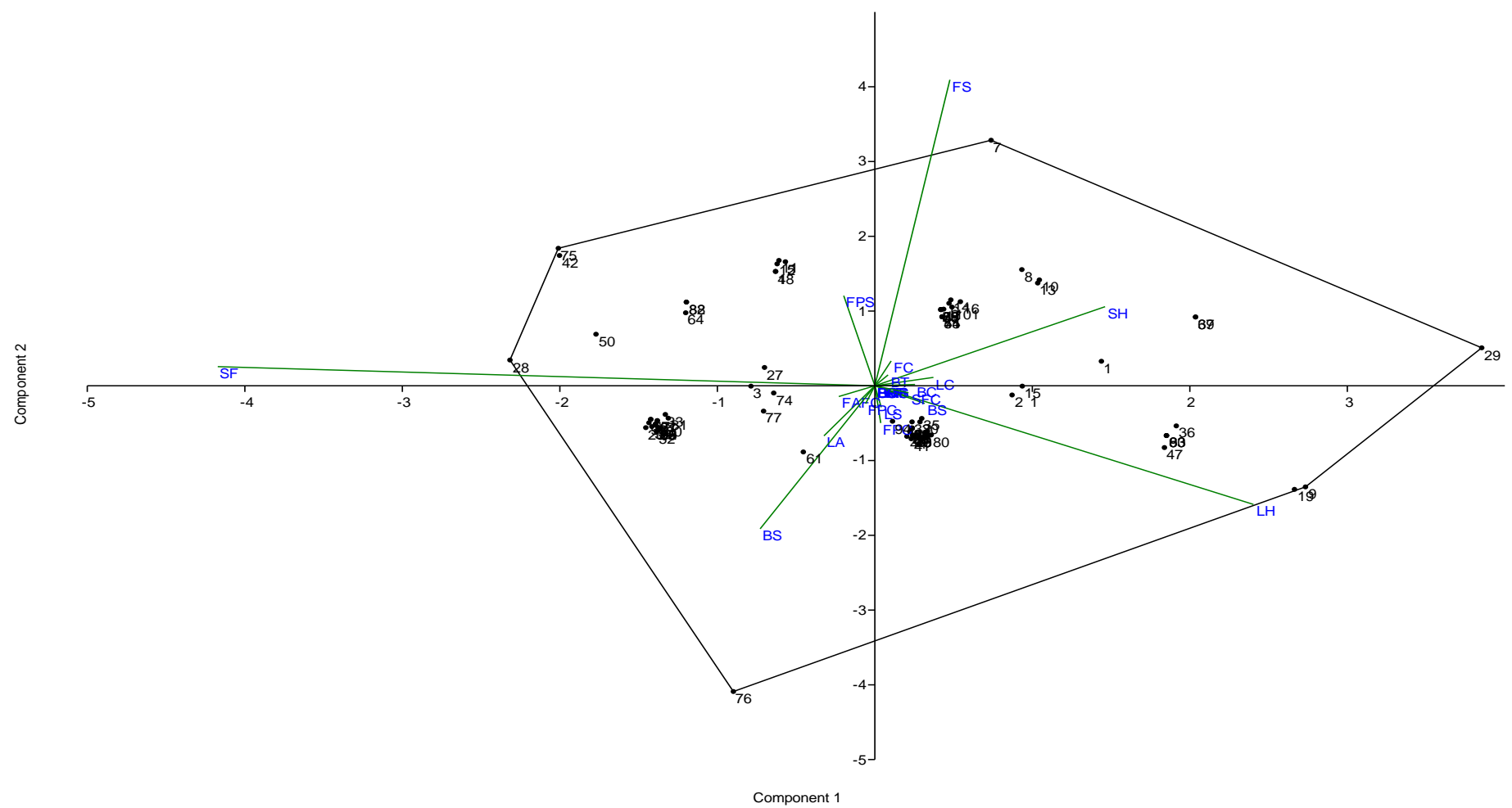

Fig.2 Two dimensional ordinations of 101 germplasm lines of upland cotton

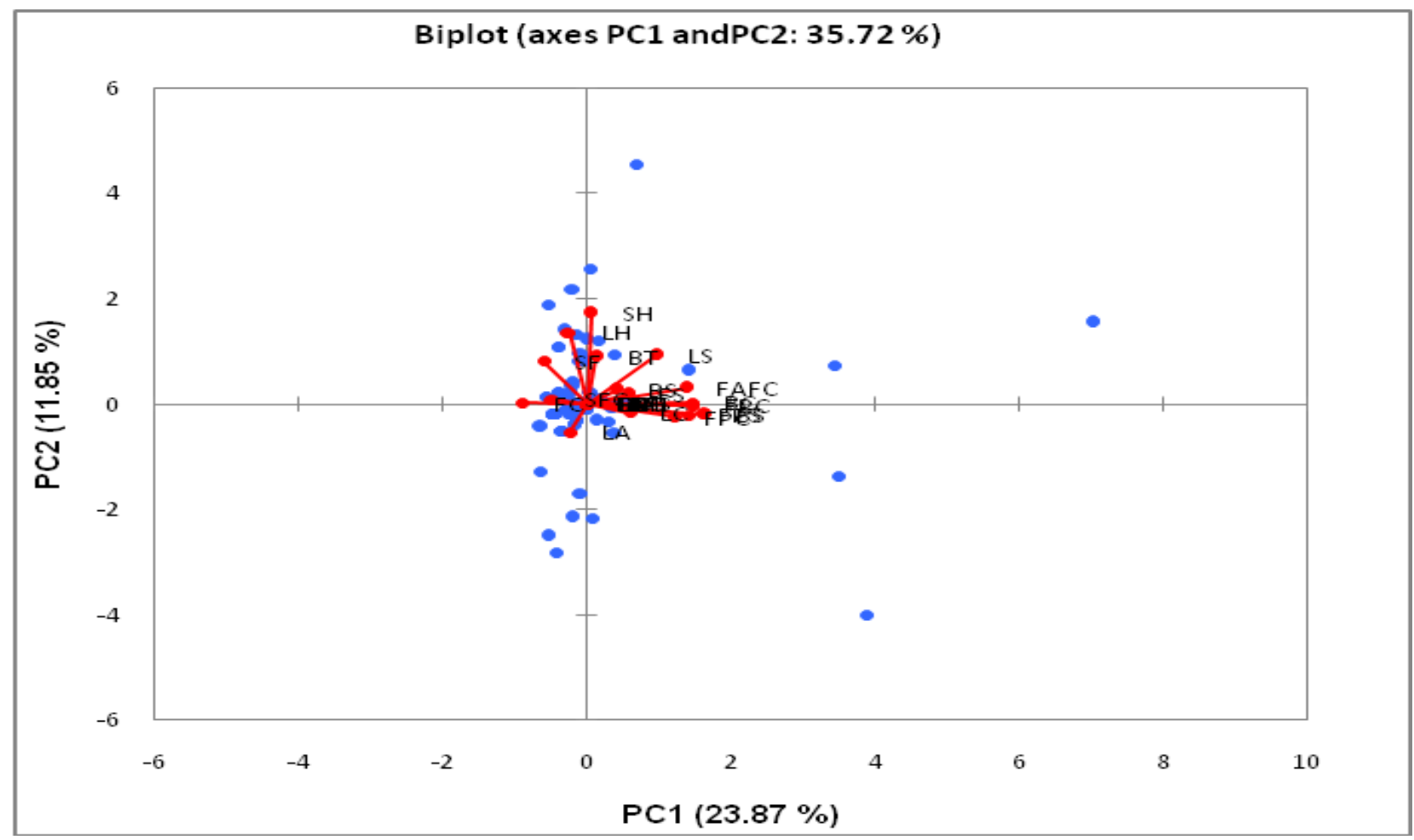


Fig.3 Dendrogram based on Ward's linkage method of qualitative characters

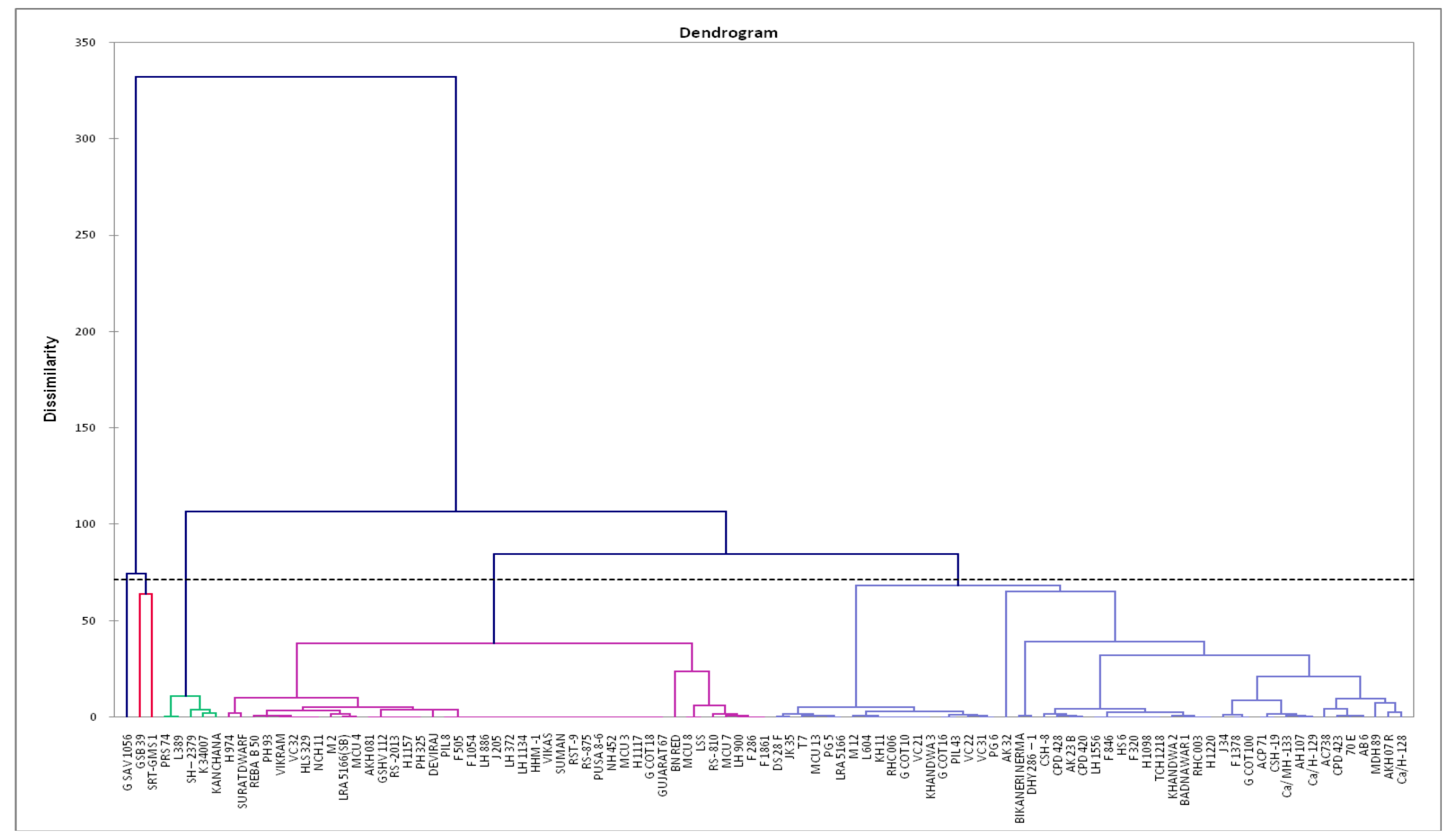


The traits like boll surface, boll colour, petal colour, petal spot, anther filament colour, pollen colour and leaf shape showed considerably positive factor loadings on PC I while leaf hairiness and leaf appearance showed negative loadings. The $2^{\text {nd }} \mathrm{PC}$ was related to diversity among cotton genotypes due to plant stem hairiness, leaf hairiness, seed fuzz, bract type and leaf shape with their positive loadings. The PC III was explained by variation due to leaf shape, leaf appearance, seed fuzz colour, and bract type with their positive loadings whereas negative loadings exhibited by leaf colour. The PC IV was elucidated diversity for leaf colour and bract type with positive loadings. The PC V exhibited variation due to bract type and flower stigma. The PC VI was related to diversity due to seed fuzz colour, flower stigma and fibre colour. The PC VII was related to diversity for boll shape and leaf appearance.

Unlike quantitative traits, qualitative traits are widely preferred for morphological characterization of the germplasm, because they are relatively less influenced by the environment. They form discrete phenotypic classes and therefore highly useful tool for classifying genotypes that are predominantly assessed visually, even by novice evaluators. Thus, in large numbers of crops DUS (distinctiveness, uniformity and stability) descriptors for plant varieties are defined using qualitative traits. However, their numbers are often limited and the spectrum of variability are relatively low (Kruskal 1978). On the other hand Ghafoor et al., (2001) showed multivariate analyses would be the valid system to deal with germplasm evaluation.

\section{Grouping genotypes based on PCA biplot}

A scatter plot (Fig. 1) was drawn using PC1 and PC2 factor scores (Table 4). A clear pattern of grouping of genotypes was observed in the factor plane. Convex of the hull occupied by the genotypes namely L389, PRS 74, Kanchana, BN Red, Bikanari Nerma, DHY 286-1, AK 32 and SRT-GMS1 and also showed the highest point of diversity among the genotypes. These diverse genotypes can be easily distinguishable among the rest and may be used as distinct parents for further breeding programmes, especially hybridization. The genotypes AC 738 and GSAV -39 held in proximity with each other which may fall in same group. The genotype GSAV 1056 separately placed in a single point indicated its distinctness. The other genotypes are congregated in a single position in the pane.

In the PC biplot, variables and genotypes are super imposed as vectors. The relative length of the vector represents the proportion of variability existed in each variable. The varieties which were far away from the origin showed more diversity with less similarity over other varieties.

The distance of each variable with respect to PC-1 and PC-2 showed the contribution of these variables towards the variation of genotypes studied. High amount of variation observed for stem hairiness, leaf hairiness, bract type, leaf shape, anther filament colour, pollen colour, petal colour, boll shape, boll colour and petal spot. The traits fibre colour and leaf appearance is not in desirable direction. The remaining traits exhibited least variability (Fig. 2). The variability among the traits studied exhibited divergently across the genotypes which can be utilized effectively.

\section{Cluster analysis}

The factors corresponding to $17 \mathrm{PCs}$ were subjected to cluster analysis based on Agglomerative hierarchical clustering performed on the Euclidean distance matrix 
utilizing the Ward's linkage method and the resultant dendrogram is presented in figure 3. The dendrogram showed five distinct clusters. The cluster II is the largest, comprising 57 genotypes followed by cluster I, which accommodate 36 genotypes. Rest of the genotypes are occupied by clusters III, IV and $\mathrm{V}$ each containing five, two and one genotype respectively. The cluster $\mathrm{V}$ is a solitary occupying the genotype GSAV 1056 (Table 4). Therefore, this genotype can be directly used as a morphological marker. May et al., (1995) reported that cluster analysis identified groups of cotton cultivars those were more closely related.

The cluster I is contributed maximum by leaf hairiness (LH) and bract type (BT). Similarly, the cluster II comprised of genotypes having the highest value and reasonable values of leaf colour (LC). None of the traits contributed towards cluster III as has been exhibited. The genotypes in cluster IV showed higher values of boll surface (BS), boll shape (BSh) and petal colour. The cluster $\mathrm{V}$ is characterised maximum by boll surface (BS), anther filament colour (FAFC), petal spot (FPS), stem hairiness (SH), boll colour (BC), position of stigma (FS) (Table 5).

Higher level of dissimilarity was found between the pairs of genotype namely Kanchana and GSAV 1056 (15.75); F 1378 and GSAV 1056 (14.697); G.cot.18 and GSAV 1056 (14.697); F 1378 and GSB 39 (14.49); G.cot. 18 and GSB 39(13.93); Kanchana and GSB 39 (13.34); F 1378 and Kanchana (5.66); G.cot.18 and Kanchana (4.899); F 1378 and G.cot.18 (2.83). However, the remaining pairs of genotype were found to possess zero dissimilarity indices. It is clearly evident that genotypes Kanchana, F 1378 and G.cot.18 were quite different from GSAV 1056 and GSB 39. The divergent genotypes can be used for hybridisation programme.
It was reported that cluster analysis had the singular efficacy and ability to identify accessions with the highest level of similarity using dendrogram (Aliyu et al., 2000). Pillay and Myers (1999) and Abdukarimov et al., (2003) have observed low genetic diversity in cotton while employing cluster analysis.

\section{Shannon-Weaver diversity index (H')}

The H' of 26 qualitative traits was used for estimating the frequency distribution of diversity index (Table 6). The H' of the traits hypocotyl pigmentation, gossypol glands, leaf nectarines, petiole pigmentation, stem pigmentation, male sterility, boll bearing habit, boll prominence of tip and boll opening were higher (4.62). An average index $\left(\mathrm{H}^{\prime}\right)$ of 4.58 was observed for 26 qualitative traits.

In conclusion, the $\mathrm{PC}$ analysis, cluster analysis and correlation coefficient in the present set of experimental data facilitated classification of genotypes. The principal component analysis, which reduces the number of traits, is in accordance with the results of cluster analysis and correlation analysis of the characteristics. The genotype GSAV 1056 having semi digitate leaf shape, presence of petal spot, anther filament colour, red coloured boll and pitted boll surface form a solitary cluster.

Therefore, this genotype can be used for future breeding programme. These distinct morphological traits can be useful for maintaining the varietal purity and aid to identify the presence off-types. In the era of Intellectual Property Rights, and Plant Breeder's Right's, qualitative morphological traits can be used as DUS (Distinctiveness, Uniformity and Stability) characters for Plant Variety Protection. In conclusion, these study clearly emphasis the use of morphological markers in characterization and utilization of plant genetic resources of cotton. 


\section{References}

Abdukarimov AS, Djataev S, Abdukarimov I (2003) Cotton Research in Uzbekistan: Elite Varieties and Future Cotton Breeding. Proceedings of World Cotton Research Conference, Cape Town, 27 September 2004, 5-15.

Akter A, Hasan MJ, Paul AK, Mutlib MM, Hossain MK (2009) Selection of parent for improvement of restorer line in rice (Oryzasativa L.). SAARC Journal of Agriculture 7:43-50.

Aliyu BN, Fawal Q, Kohel RJ (2000) Inheritance of Pubescence in Crosses between $V$. unguiculata and $V$. rhomboidea. Nigerian Journal of Genetics, 15, 9-14.

Chen ZJ, Scheffler B E, Dennis E, Triplett B, Zhang $\mathrm{T}$, Chen $\mathrm{X}$, Stelly DM, Rabinowicz PD, Town C, Arioli T, Brubaker C, Cantrell R, Lacape JM, Ulloa M, Chee P, Gingle AR, Haigler C H, Percy R, Saha S, Wilkins T, Wright RJ, Deynze AV, Zhu Y, Yu S, Guo W, Abdurakhmonov I, Katageri I, Rahman M, Zafar Y, Yu JZ, Kohel RJ, Wendel J, Paterson AH (2007) Towards sequencing cotton (Gossypium) genomes. Plant Physiol. 145: 13031310.

Dutt Y, Wang XD, Zhu YG, Li YY (2004) Breeding for high yield and fibre quality in coloured cotton. Plant Breed. 123:145-151.

Esmail RM, Zhang JF, Abdel-Hamid AM (2008) Genetic diversity in elite cotton germplasm lines using field performance and RAPD markers. World Journal of Agriculture Science 4: 369375.

Ghafoor A, Sharif A, Ahmad Z, Zahid MA, Rabbani MA (2001) Genetic Diversity in Black Gram (Vigna mungo L.). Field Crops Research, 69:183-190. http://dx.doi.org/10.1016/S0378-
4290(00)00141-6

Hammer O, Harper DAT, Ryan PD (2001) PAST: Paleontological statistics software package for education and data analysis. Palaeontol. Electr. 4: 1-9

Hosseini G (2014) Estimation of genetic parameters for quantitative and qualitative traits in cotton cultivars (Gossypium hirsutum L. and Gossypium barbadense L.) and new scaling test of additive- dominance model. Journal of Plant Molecular Breeding, 2(1): 45-63.

Iqbal MJ, Aziz N, Saeed NA, Zafar Y (1997) Genetic diversity evaluation of some elite cotton varieties by RAPD analysis. Theore. Appl. Genet. 94:139-14.

Kruskal, JB (1978) Factor analysis and principal component analysis: bilinear methods. In: Kruskal WH, Tannur JM (eds) International encyclopedia of statistics. The Free Press, New York, pp 307-330.

Kumar S, Joshi UN, Singh V, Singh JV, Saini ML (2013) Characterization of released and elite genotypes of guar (Cyamopsis tetragonoloba (L.) Taub.) From India proves unrelated to geographical origin. Genet Resour Crop Evol. doi:10.1007/s10722-013-9970-7

Li Z, Wang X, Yan Z, Guiyin Z, Wu L, Jina C, MA Z (2008) Assessment of genetic diversity in glandless cotton germplasm resources by using agronomic traits and molecular markers. Frontiers of Agriculture in China 2: 245-252.

May OL, Bowman DT and Ca lhoun DS (1995). Genetic diversity of US upland cotton cultivars released between 1980 and 1990. Crop Sci., 35: 1570-1574.

Meredith WR Jr. (2000) Cotton yield progress- why has it reached a plateau. Better Crops 84:6-9.

Pearson K (1901) On lines and planes of closest fit to systems of points in space. Philos Mag A. 6:559-572.

Plantauthority.gov.in 
Pecetti L, Annicchiario P, Damania AB (1996) Geographic variation in tetraploid wheat (Triticum turgidum spp. Turgidumconvar. Durum) landraces from two provinces in Ethiopia. Genetic Resources and Crop Evolution 43:395-407.

Pillay M, Myers GO (1999) Genetic Diversity in Cotton Assessed by Variation in Ribosomal RNA Genes and AFLP Markers. Crop Science, 39:1881-1886. http://dx.doi.org/10.2135/cropsci1999.3 961881x

Saravanan S, Arutchendhil P, Raveendran TS, Koodalimgam K (2006) Assessment of genetic divergence among introgressed culture of Gossypium hirsutum L. through RAPD analysis. J. Applied Sci., Res., 2: 1212-1216.

Shakeel A, Farooq J, Ali MA, Riaz M, Faooq

A, Saeed A, Saleem MF (2011) Inheritance pattern of earliness in cotton (Gossypium hirsutum L.). Aust. J. Crop Sci. 5:1224-1231.

Shannon CE, Weaver W (1949). The mathematical theory of communication. Univ. of Illinois Press, Urbana.

Sharma JR (1998) Statistical and Biometrical Techniques in Plant Breeding. New Age International (P) Limited Publishers, New Delhi. 432.

Sneath PHA, Sokal RR (1973) Numerical Taxonomy: The Principles and practice of numerical classification. Free-Man WF and Co, San Francisco, USA.

Van Hintum TJL (1995) Hierarchical approaches to the analysis of genetic diversity in crop plants. In: Hodgkin, T., Brown, A.H.D., Van Hintum T.J.L., Morales, E.A.V. (Eds.) Core collection of plant genetic resources. John Wiley and Sons, pp. 23-34.

Ward JH (1963) Hierarchical grouping to optimize an objective function. J. Am. Stat. Assoc. 58:236 -244.

Zada M, Zakir N, Rabbani MA, Shinwari ZA (2013) Assessment of genetic variation in Ethiopian mustard (Brassica carinata A. Braun) germplasm using multivariate techniques. Pak J Bot 45(S1):583-593.

\section{How to cite this article:}

Rathinavel, K. 2017. Exploration of Genetic Diversity for Qualitative Traits among the Extant Upland Cotton (Gossypium hirsutum L.) Varieties and Parental Lines. Int.J.Curr.Microbiol.App.Sci. 6(8): 2407-2421. doi: https://doi.org/10.20546/ijcmas.2017.608.285 